\title{
La United Fruit Company y su relación con las zonas bananeras en países del Mar Caribe y Caribe colombiano
}

\author{
Maribel de la Cruz Vergara \\ Universidad de Cartagena \\ mdelacruzv@unicartagena.edu.co
}

Recibido: octubre de 2014

Aprobado: febrero de 2015

Resumen: Con este trabajo se muestran las relaciones que estableció la United Fruit Company en las zonas bananeras de los países del Mar Caribe y el Caribe colombiano; así mismo la participación de esta multinacional en la explotación de materias primas, vista desde la comparación entre países. En consecuencia se abordan algunos de los problemas trasnacionales que este trust (United Fruit Company) creo y tuvo que afrontar en estas zonas bananeras, destacándose su participación directa en los conflictos entre fronteras, contratos y producción.

Palabras claves: Bananeras, Colombia, Mar Caribe, United Fruit Company, conflictos.

\section{The United Fruit Company and its relation to the banana plantations in countries of the Caribbean Sea and the Colombian Caribbean}

Abstract: With this work the relationships established between the United Fruit Company in the banana plantations in the countries of the Caribbean Sea and the Colombian Caribbean are shown; likewise the participation of this multinational in

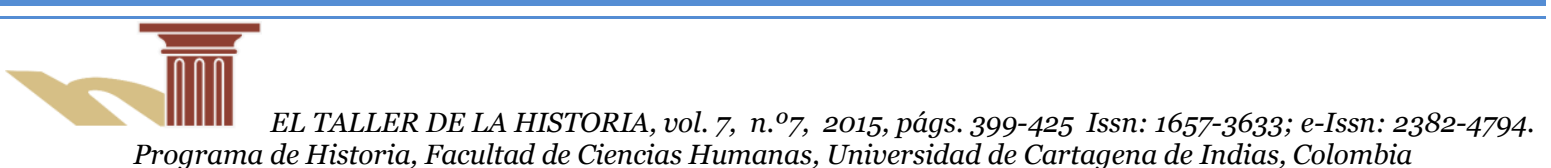


the exploitation of raw material, seen from the comparison between countries. In consequence some of the transnational problems that this trust (United Fruit Company) created and had to confront in this banana plantations are presented, highlighting its direct participation in the conflicts between borders, contracts and production.

Key words: Banana plantations, Colombia, Caribbean Sea, United Fruit Company, conflicts.

\section{Introducción}

A finales del siglo XIX la era del control inglés sobre las economías latinoamericanas había llegado a su fin, mientras que Estados Unidos se constituyó en una de las fuerzas industriales más importante del mundo, por lo que buscó incursionar en los nuevos mercados de Asia, África y América Latina, siendo este último el mercado predilecto para la inversión de capital en la producción de materias primas. Si bien es cierto que en 1885 el comercio de este país con América Latina constituía solamente el 3,74\% de sus exportaciones, también lo es que visto de más de cerca, este era predominante en algunos países de la región. Así controlaban el $64 \%$ del comercio de Guatemala; el 41,6\% del de Venezuela; el 39,4 \% del de México; el 36,6\% del de Colombia y el $26,8 \%$ del brasileño. ${ }^{1}$

El comercio desigual con Estados Unidos abarco todos los renglones de producción de materia prima, sin embargo la instauración y explotación de las zonas bananeras se convirtió en un tema, que desde comienzos del siglo xx y con la consolidación de la United Fruit Company, tuvo muchas críticas tanto por los demás

\footnotetext{
${ }^{1}$ Hugo Murillo Jiménez et al, "Las relaciones comerciales entre Costa Rica y los Estados Unidos a través de los informes consulares, 1880-1901", en Anuario de Estudios Centroamericanos vol.13, n. ${ }^{\circ}$, San José de Costa Rica, Universidad de Costa Rica, 1978, pp.135-136.
} 
productores norteamericanos en el negocio, a los que esta Compañía saboteo y sacó deslealmente del mercado, como los pequeños productores latinoamericanos y trabajadores en general.

De lo que se trata en esta investigación es traer nuevamente a la discusión, el papel de la intervención norteamericana en los países de America Latina y el Caribe, la articulación de estos en su economía y las tendencias de desarrollo que se establecieron a partir de dicha intervención. Esto visto más allá del discurso de la dependencia que nos permita en la actualidad reflexionar alrededor de las articulaciones que se han ido estableciendo en nuestra región a través de los Tratados de Libre Comercio.

Para lo cual analizaremos el establecimiento de la United Fruit Company en las zonas bananeras de Colombia, Costa Rica y Honduras, para determinar puntos comunes de la producción bananera, de las políticas públicas y los conflictos, fronterizos suscitados por la explotación en estos países.

\section{Establecimiento de la producción bananera a gran escala en Colombia}

El afán de levantar la economía por la guerra de los mil días ${ }^{2}$ y sobre todo por la crisis que atravesaba el café, (cuya taza de crecimiento anual decayó en un 3,3\% entre 1874 y 1898 , y en 2,9\% anual, comparando el año de 1874 momento de auge con el de 1913) ${ }^{3}$ cuyos precios de exportación habían caído en el mercado internacional a finales del siglo XIX y primeros años del XX, afecto a otros sectores de la

\footnotetext{
2 Jesús Antonio Bejarano Ávila, "El despegue cafetero (1900-1928)", en Historia Económica de Colombia, Bogotá Editorial Planeta, 2007, pp.195-232. Véase también a Salomón Kalmanovitz y Enrique López Enciso, La agricultura Colombiana en el siglo XIX, Bogotá, Fondo de Cultura Económica, 2006; Bernardo Tovar Zambrano, La intervención económica del Estado en Colombia, 1914-1936, Bogotá Banco Popular, 1984; Arnold Bauer, "La Hispanoamérica rural, 1870-1930", en Historia económica de América latina, Madrid, Crítica, 2002; Willian Glade, "América Latina y la economía internacional, 1870-1914”, en Historia económica de América Latina.
}

${ }^{3}$ Willian Glade, “América Latina y la economía internacional, 1870-1914”. 
economía, como el sector ganadero y el de la caña de azúcar, ${ }^{4}$ debido a la baja en el consumo de la carne, por la que los trabajadores pagaban altos precios, y al bajo consumo de alcohol por la perdida del poder adquisitivo de estos, se convirtió en uno de los factores fundamentales para estimular otros productos agrícolas dentro de los que el banano fue el más viable por el bajo costo en su producción y la demanda creciente en el exterior.

Así en 1908, cuando Rafael Reyes ${ }^{5}$ presidente de Colombia leyó frente a los diplomáticos, comerciantes y agricultores su discurso en donde justificaba la importancia del "trabajo honrado, inteligente y ordenado para el bienestar económico del país”, y de la importancia de crear una gran zona geográfica para el desarrollo de la producción bananera a gran escala, estaba lejos de vislumbrar que con esta acción daba inició a la intervención y control de la United Fruit Company en la producción bananera, y sus posteriores consecuencias económicas y sociales.

Realizar los estudios pertinentes, tanto desde el punto de vista económico como geográfico, se convirtió entonces en una de las prioridades del gobierno, por lo que se asignó tal tarea a los Generales Perdomo y Calderón, quienes con las autoridades del departamento del Magdalena, el representante de la United Fruit Company, el Gerente del ferrocarril de Santa Marta, ${ }^{6}$ realizaron esta exploración, es decir, tanto en la visita como en el aval para la iniciación del negocio estuvieron involucradas todas las autoridades requeridas.

De otra parte, se consideraba que había ciertas condiciones propicias para iniciar tal empresa, dentro de las cuales se contaban:

\footnotetext{
${ }^{4}$ Rafael Reyes, Conferencia leída el 19 de abril de 1908 en el Salón de Grados, Colombia, Imprenta Nacional, Bogotá, 1908, p.8

5 R. Reyes, Conferencia leída el 19 de abril de 1908 en el Salón de Grados.

${ }^{6}$ Participaron el ex Gobernador del departamento Dr. Ramón Goenaga, quien era uno de los más interesados en el negocio, por la United Fruit Company, Mr. M. F. Carr, el gerente del ferrocarril de Santa Marta Mr. P Marschal, Mr Brandbury, el Secretario General de la gobernación Dr. Emiliano J. Gálvez, por el Dr. José F. Torres, alcalde de Ciénega.
} 
a) Muchas personas con capacidad empresarial y capital, que producto de la guerra de los mil días se habían establecido en otros países en donde fundaron negocios prósperos, en este punto se consideraba que se tenía el capital humano y económico para el desarrollo de la industria nacional. Dentro de estos se contaban los Duran, Pino, Díaz, Eraso, Ossa, Díaz Granados, Arosemena, Calvo, Gómez Valdéz, Orrantía, Gonzáles Tello, quienes emigraron hacía Guayaquil en donde desarrollaron el cultivo del cacao, el comercio y la banca. Los Ospina, Vásquez, Álvarez, Recaredo de Villa, Párraga, quienes desarrollaron el comercio, la agricultura y la banca en Guatemala y El Salvador; los Ribón, Muñoz, De Castro, Espriella, Camacho Roldan, Samper, Saenz, Jiménez quienes habían fundado negocios en New York. Los Díaz Granados, Zubiría, García, Vengoechea, quienes se establecieron en París como comerciantes y negociantes, e igualmente y bajo las mismas condiciones se establecieron en Londres Los Montoya, Saénz, Merino, Cortés y Parga.

Debido a que estos empresarios exitosos habían emigrado y a que se consideraba la capacidad empresarial hereditaria económica y socialmente, se contaba con que sus descendientes eran los llamados a replicar dicho esquema en el país, expresado por Rafael Reyes en los siguientes términos: "Los descendientes de estos obreros de la civilización y del progreso existen hoy en todo el país; en este mismo recinto hay muchos de ellos, y todos comprenden que su deber es no emigrar, como sus antepasados se vieron obligados a hacerlo, sino a la sombra bienhechora de la paz emplear sus capacidades y energías para hacer grande y feliz la tierra de sus padres".7

b) había que aprovechar los tiempos de paz, para lograr tal cometido, ya que las guerras del siglo XIX habían diezmado la economía.

c) el mercado estaba ofreciendo las condiciones para que se desarrollara la producción bananera, ya que el consumo interno en Estados Unidos había

\footnotetext{
${ }^{7}$ R. Reyes, Conferencia leída el 19 de abril de 1908 en el Salón de Grados, p.4.
} 
crecido, mientras Europa se convertía en un mercado atractivo debido a que mostraba una tendencia al incremento del consumo de esta fruta;

d) la United Fruit Company tenía presencia en casi todos los países del Caribe;

e) los terrenos que recorría el ferrocarril de Santa Marta, eran aptos y adecuados para el desarrollo del producto, además que ya se contaba con la infraestructura misma lo que facilitaría el negocio.

f) las enfermedades como la fiebre amarilla y el paludismo propias de estas regiones ya estaba controladas.

g) Se contaba con el mejor puerto de las antillas, en donde podrían atracar grandes buques sin necesidad de muelle, así como el inicio del ferrocarril el cual recorría toda la zona

h) En estos mismos terrenos también se podía estimular el cultivo del caucho y cacao y por último

i) como símbolo del entusiasmo y el afán de progreso, mediante el cual se pretendía rebasar el desarrollo de México y Argentina, se planteó establecer en Cartagena un centro de acopio o bodega de almacenamiento de pasto, es decir una Packing House, mejor que las existentes en dichos países.

Las bondades de la United Fruit Company y la industria bananera en el Magdalena. El negocio del banano sonaba atractivo no solamente por el incremento en su consumo en el exterior sino también porque el trust que tenía el monopolio se vendía como una de las formas que América Latina y el Caribe tenían para jalonar su propio desarrollo, de tal manera que en los años de 1906 y 1907, la empresa tuvo excelentes utilidades, así como el control del 90\% del banano que se importaba por Estados Unidos y exportaban para Europa, ejerciendo el control del precio. Sus plantaciones estaban en Costa Rica, Cuba, Santo Domingo, Jamaica, Honduras, Guatemala, Panamá, Guayana Holandesa entre otros. Contaba además 
con una flota de vapores propia con la que sacaban la producción de las zonas bananeras, además del control del ferrocarril de los países en donde se establecía.

En el balance de este bienio la Compañía mostró un capital de 32.721.183,14 pesos oro americano y utilidades en la producción e importación de banano en el año 1906 \$3.720.402,41 y en el año 1907 \$6.061.910,42, mostrando un incremento en ganancias de $\$ 2.341 .508,01$, correspondiente al $62 \%$, de otra parte el crecimiento total de cada período fiscal comparando el año 1 con relación al año 2 fue del 76,54\%, de tal manera que de acuerdo con los balances presentados por la Compañía el gobierno consideró que había llegado el momento para que el país entrara a la era del desarrollo (ver grafica 1).

Grafica 1. Comparativo de las utilidades en los años fiscales 1906 y 1907 , The United Fruit Company

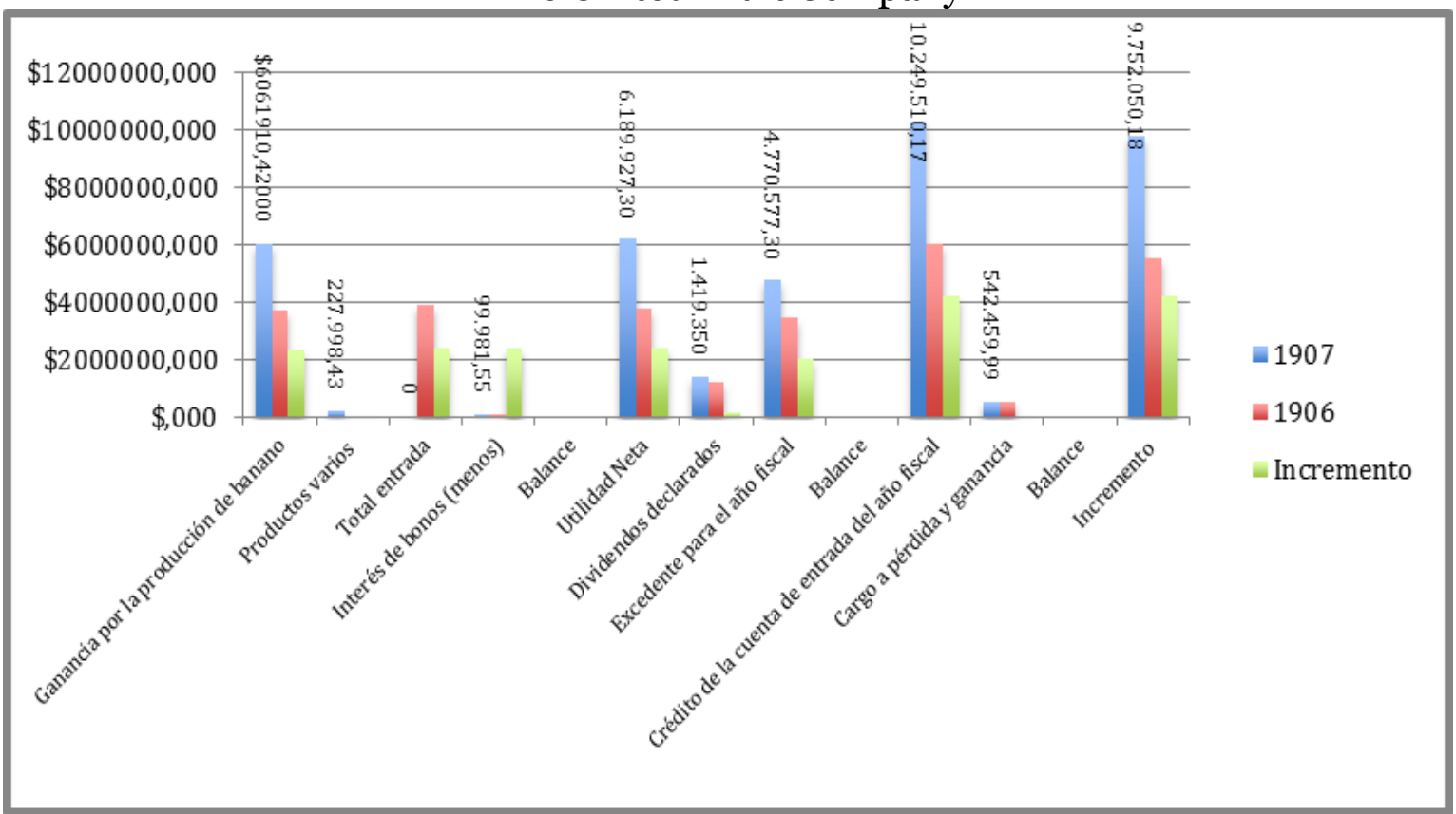

Fuente: Rafael Reyes. Conferencia leída el 19 de abril de 1908 en el salón de grados, p. 11, elaboración de la autora.

El trust, además del banano mostraba interés en la producción de otros productos agrícolas, tales como naranja, piña, plátano caña de azúcar, cocos, pastos 
que ya se cultivaban por los campesinos y productores locales, ${ }^{8}$ además del guineo Papares que se cultivaba en la finca de propiedad de la familia Mier, ${ }^{9}$ con el fin de lograr o superar la producción de Centro América e Islas de Caribe.

El departamento del Magdalena, ${ }^{10}$ fue seleccionado para el desarrollo de la zona bananera por sus condiciones geográficas y climáticas, también se tuvo en cuenta el hecho de que el río Magdalena se convertía en corredor natural en el cual se asentaban aproximadamente 500.000 habitantes, de los cuales se podía captar mano de obra barata y con capacidad de adaptarse a las condiciones climáticas, estos habitantes se contaban desde Barranquilla hasta Neiva, en total para los años señalados anteriormente, se contrató aproximadamente 15.000 trabajadores los que procedían en su mayoría del departamento de Bolívar.

Otro aspecto que hizo posible esta zona, encuentra sus fundamentos en que el banano que se producía en la región del Magdalena, era de mejor calidad que el de las plantaciones de Costa Rica, Panamá, Jamaica, Guatemala y Santo Domingo, dichas zonas eran controladas por la United, tanto en la producción como en el precio; los ríos con abundante agua como Río Frío, Sevilla y Fundación permitían el cultivo a bajo costo; en cuanto a las tierras se contaba con que estas pertenecían a la nación por lo que no se afectaba ningún particular, condiciones que permitieron trazar la zona bananera entre Santa Marta y Fundación. La conexión entre la línea del ferrocarril (de más de 90 kilómetros) y el puerto también se convirtió en otra de las ventajas de la Zona (véase mapa 1).

\footnotetext{
${ }^{8}$ Sobre el desarrollo y crecimiento económico de Santa Marta antes del establecimiento de la zona véase a Jorge Enrique Elías Caro, "El puerto de Santa Marta: determinantes de crecimiento y desarrollo de una ciudad caribeña (1810-1860)", en Ciudades portuarios en la Gran Cuenca del Caribe, Visión histórica, Barranquilla, Universidad del Norte/Universidad del Magdalena, 2010, pp.328354

${ }^{9}$ R. Reyes, Conferencia leída el 19 de abril de 1908 en el Salón de Grados, p.14

${ }^{10}$ Adolfo Meisel Roca, "Enfermedad Holandesa y exportaciones de banano en el caribe colombiano, 1910-1950", en Cuaderno de Historia económica y Empresarial, Cartagena, Banco de la República, 2010, pp.1-56.
} 


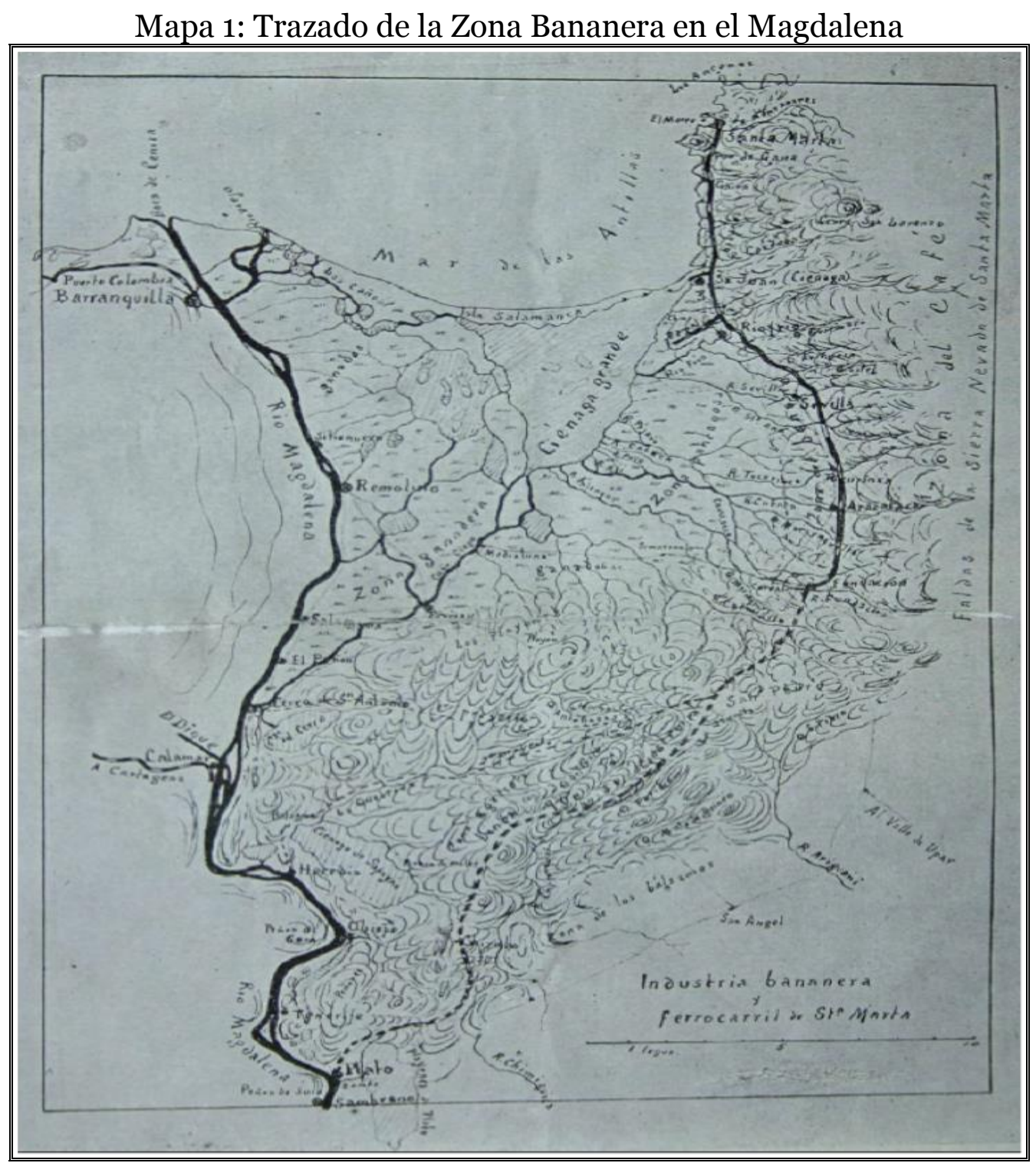

Fuente: Rafael Reyes, Conferencia leída el 19 de abril de 1908 en el salón de grados.

De otra parte, según el gobierno la industria bananera y productos relacionados, incluyendo el procesamiento del algodón en Medellín, permitirían el equilibrio económico del país, así como la ocupación de productores, comerciantes y trabajadores en esta actividad, coadyuvaría a mantener la paz, por lo que se hizo un 
llamado a los conciudadanos para que se dedicaran a esta y otras industrias "[...] con el mismo mayor entusiasmo y fervor que en otro tiempo se aplicaban a fomentar revoluciones y matanzas entre hermanos". ${ }^{11}$

Con el fin de estimular las iniciativas nacionales se calculó la rentabilidad del negocio para los interesados en 100 hectáreas de tierras baldías, ${ }^{12}$ para iniciar una plantación, la cual según cálculos del gobierno para que produjeran se necesitaba una inversión de $\$ 100$ oro, por lo que 100 hectáreas costarían $\$ 10.000$ oro, dando una rentabilidad total de $\$ 8.000$ oro, por lo que en el primer año de trabajo se pagaría el costo de la misma, estos productores venderían directamente a United Fruit Company, quien sería la encargada de la exportación del banano; ya desde esa época hubo algunas voces advirtiendo que los pequeños productores y el Trust no eran una buena formula, pues de alguna manera competirían en la producción del mismo fruto.

La huelga general de trabajadores en 1928 y el incumplimiento de la Compañía The Santa Marta Railway Company Limited, filial de la United Fruit Company, no cumplió con parte del contrato, en lo referente a la construcción del ferrocarril y con la puesta en marcha del tren al servicio del público, por lo que la Corte Suprema de Justicia de Colombia, aclaró en primer lugar que el ferrocarril del Magdalena le pertenecía a la Nación, estableció las sanciones correspondientes tanto por el incumplimiento del contrato como por los perjuicios causados al departamento del Magdalena, además de vender el ferrocarril preferentemente a la Nación

De otra parte en Colombia se sumó a los problemas legales, los de la huelga de trabajadores, ${ }^{13}$ los que demandaban:

\footnotetext{
${ }^{11}$ R. Reyes, Conferencia leída el 19 de abril de 1908 en el Salón de Grados, p.10.

12 Amparados en la ley 48 de 1882 sobre tierras baldías, en la cual se determinó que las tierras baldías se podían adquirir solo por cultivo, cualquiera fuera su extensión, véase en Luis Fernando López Garavito, Historia de la Hacienda y el tesoro en Colombia, 1821-19oo, Bogotá, Banco de la República, 1992, p.283.

${ }^{13}$ Sobre, la huelga en las bananeras y masacre contra los trabajadores y problemas sociales generalizados relacionados con esta véase entre otros a: Carlos Arango Zuluaga, Sobrevivientes de las bananeras, Bogotá, Ecoe, 1985; Mauricio Archila Neira, "Masacre de las bananeras: diciembre 6 de
} 
- Seguro colectivo y obligatorio para obreros y empleados de la empresa de acuerdo con las leyes 37 de 1921 y 32 de 1922.

- Cumplimiento de la ley 57 de 1925 sobre accidentes laborales de los trabajadores de la empresa y los agricultores que de ella dependen.

- Cumplimiento de la ley 46 de 1918 sobre habitaciones higiénicas, de la ley 15 de 1925 sobre higiene social y asistencia pública y de la ley 76 de 1926 sobre descanso dominical.

- Aumento en un 50\% de los jornales de los empleados que ganan menos de 100 pesos.

- Cesación de los comisariatos o establecimientos comerciales de artículos de primera necesidad de la United Fruit Co, y establecimiento del libre comercio en la zona bananera.

- Cesación de préstamos por medio de vales.

- Cesación de pagos por quincena y establecimientos de estos por semanas vencida.

- Cesación de los contratos individuales y establecimiento de la contratación colectiva.

1928”, en Revista Credencial Historia n. ${ }^{\circ} 117$, Bogotá, 1999, p.5; Fernando Botero, "El enclave agrícola en la zona bananera de Santa Marta", en Cuadernos colombianos vol.3, n. ${ }^{\circ} 11$, Medellín, 1977, pp.312-389. Philip Maurice Brungardt, "La United Fruit Company en Colombia", en Innovar n. ${ }^{\circ}$, Bogotá, Universidad Nacional, 1995, pp.107-118; Catherine LeGrand, "Living in Macondo. Economy and culture in a United Fruit Company, banana enclave in Colombia", in Close encounters of Empire: writing the cultural history of U.s. Latin American relations, Durham, Duke University Press, 1998. Jorge Elías Caro, "La masacre obrera de 1928 en la zona bananera del Magdalena Colombia”, en Andes vol.22, n. ${ }^{\circ}$, Salta, Universidad Nacional de Salta, 2011. 
- Establecimiento de hospitales en número suficiente para que pudiera atenderse debidamente, el gran número de trabajadores que dependen de esta e higienización de los campamentos.

Producto de las anteriores solicitudes y después de fuertes conflictos la empresa negoció algunos de ellos, terminando este episodio con el asesinato de 100 huelguista por parte del ejército colombiano, ${ }^{14}$ heridos 238 , mientras las pérdidas de la Compañía ascendió a \$1.250.000,00 oro americano, noticias que se expandieron y se señalaban de referente en el Caribe Bananero.

\section{Los contratos de la United Fruit Company en Centro América, la injerencia en los asuntos interno y la motivación de conflictos en los límites entre países (Panamá- Costa Rica, Honduras Guatemala)}

Otro de los aspectos que hay que analizar de la United Fruit Co., es su injerencia en los asuntos internos de los países en donde se establecía, al intentar defender sus "derechos" obtenidos a través de las jugosas contrataciones y cesiones de derecho sobre las tierras baldías y líneas del ferrocarril ${ }^{15}$, tal como sucedió en el conflicto entre los límites de Panamá. Esta situación encuentra sus orígenes en una -franja de tierra entre estos países (se originó cuando Panamá pertenecía a Colombia, luego de la independencia el asunto pasó a dirimirse entre estos dos países) por el límite del pacífico, en donde la Compañía había trazado parte del ferrocarril por

\footnotetext{
14 Para otro enfoque sobre la producción bananera en Colombia véase a Marcelo Bucheli, "Enforcing Business Contracts in South America: The United Fruit Company and Colombian Banana Planters in the Twentieth Century. The President and Fellows of Harvard College", in The Business History Review vol.78, n. ${ }^{\circ}$ 2, 2004, pp.181-212. www.jstor.org/stable/25661863. Consultado 15/01/2013

${ }^{15}$ Sobre el ferrocarril en Costa Rica véase a Rodrigo Quesada Monge, "Ferrocarriles y crecimiento económico: el caso de la Costa Rica Railway Company, 1871-1905”, en Anuario de Estudios Centroamericanos vol.9, San José de Costa Rica, Universidad de Costa Rica, pp.87-119. www.jstor.org/stable/25661863. Consultado 15/01/2013
} 
donde se trasportaba el banano; sobre esta situación el presidente Jiménez ${ }^{16}$ de Costa Rica se pronunció en el sentido que la solución más viable era que esta cediera esos terrenos a Panamá, como gesto de paz y de la política del buen vecino. Además señaló que esas tierras eran "malas" por lo que ningún costarricense tenía propiedades allí, planteamiento que indudablemente no tuvo en cuenta que las zonas bananeras en Costa Rica y en los demás países del Caribe y America Latina se caracterizó básicamente por una amplia colonización de plantación de banano, paralela a la construcción del ferrocarril con escasa población.

La posición del presidente fue duramente criticada principalmente porque consideraban en primer lugar que este país no debía ceder sus territorios, y sobre todo porque el argumento del presidente no era suficiente para tomar tal decisión. Lo anterior no obstante tener la Compañía una importante explotación bananera en Costa Rica desde el año de 1883. En 45 años, es decir de $1883^{17}$ al primer trimestre de 1828 , la compañía había exportado ${ }^{18}$ básicamente hacía los Estado Unidos de America unos 200.000.000 racimos de banano (ver grafica 2), esto les daba un alto margen de ganancia, ya que el racimo se pagaba muy barato, de acuerdo con la siguiente escala: ${ }^{19}$

Racimo de 9 o más manos $=50$ ctvs. oro americano

Racimo de 8 manos $=37,5$ ctvs. oro americano

Racimos de 6 manos $=12,5$ ctvs oro americano

${ }^{16}$ Alfredo Saenz, The banana situatiotion in the countries of de Caribbean Sea, San José de Costa Rica, Imprenta Borrase Hermanos1928, p.XI

17 Aunque la United Fruit Company fue fundada en 1899, se cuenta su operación desde las compañías que le dieron vida, es decir la Tropical Trading and Transport Company fusionada con la Boston Fruit Company.

${ }^{18}$ Sobre las relaciones comerciales entre Costa Rica y Estados Unidos véase a Hugo Murillo Jiménez et al, "Las relaciones comerciales entre Costa Rica y los Estados Unidos a través de los informes consulares, 1880-1901", en Anuario de Estudios Centroamericanos vol.13, n. ${ }^{\circ}$ 2, San José de Costa Rica, Universidad de Costa Rica, 1978, pp.135-194. http://www.jstor.org/stable/25661931. Consultado: $15 / 01 / 2013$

${ }_{19}$ Alfredo Saenz. The banana situatiotion in the countries of de Caribbean Sea, pp.LIII y siguientes. 
Grafica 2. Exportación de racimos de bananos desde Costa Rica, 1883- 1927

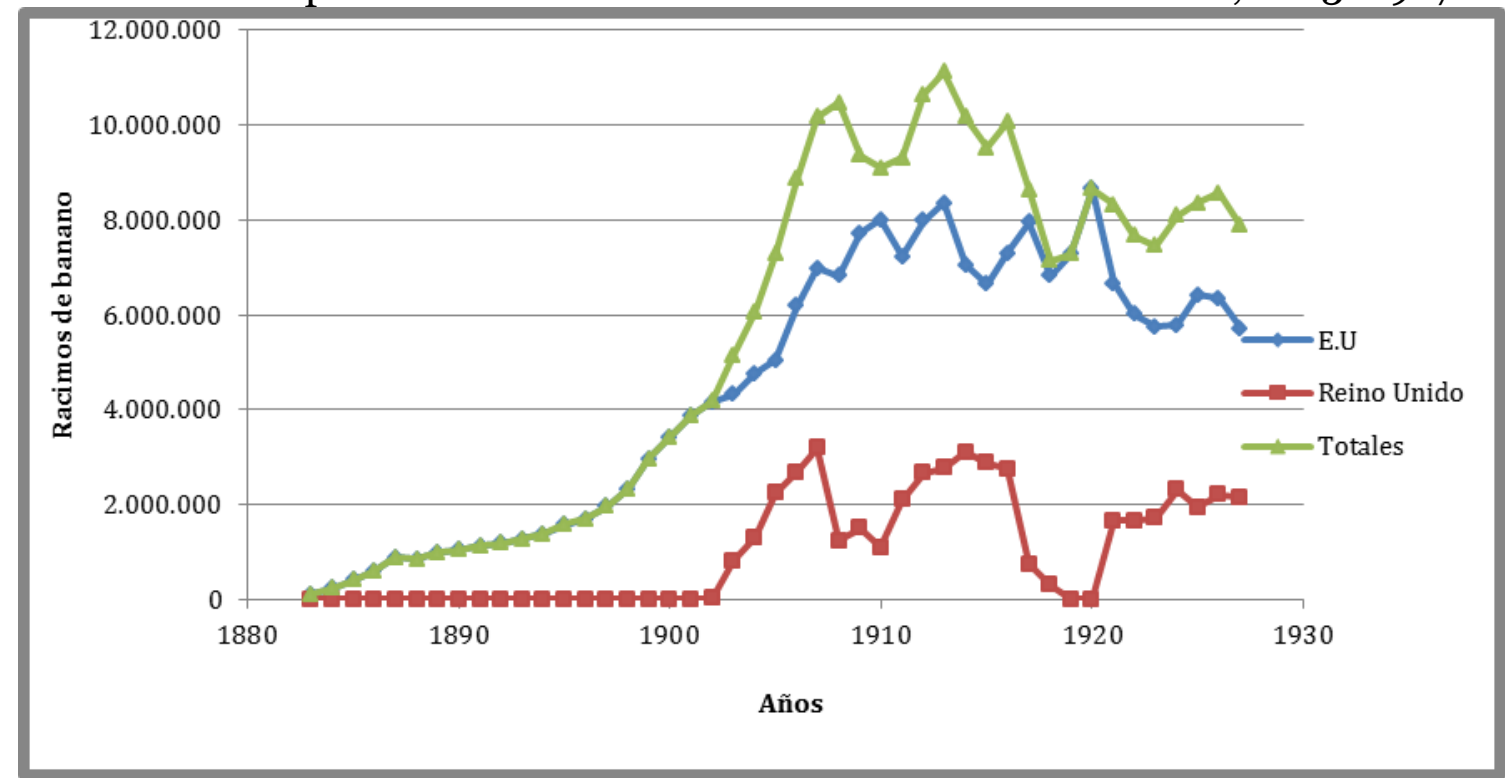

Fuente: Alfredo Saenz, The banana situation in the countries of de Caribbean Sea, pp.LIII-LIV. Elaboración de la autora

No obstante estar establecido los precios por contrato en 1919 un racimo de 9 manos se pagaba a 30 centavos, un racimo de 8 y 7 manos a 14,5 cts., mientras el racimo de 6 manos no se contaban, situación que ponía en ventaja a la United y en desventaja a los productores y trabajadores. En la gráfica 2 observamos que desde 1885 la mayoría del banano se exportaba para el mercado estadounidense y en menor medida para el Reino Unido. La exportación tuvo un periodo de auge que comenzó en 1907 hasta 1920, cuando comenzaron a cambiar las circunstancias del mercado y se avizoraba la crisis del 29.

Esto sucedía debido a que el tipo de contratos que firmaba la empresa, avalados por los gobiernos de turno, obligaba a que la producción de plantadores y contratistas debía ser vendidas a este monopolio, el cual fijaba el precio y establecía las condiciones del producto para exportación, por ejemplo para exportar solo se consideraban los racimos que tuvieran 7, 8 o 9 manos, además que los bananos debían estar verdes y limpios, sin magulladuras, sin manchas, dañados o quemados 
por el sol, para lograr estos estándares de calidad la Compañía determinaba el momento en que había que cortar el banano.

Este se hacía en promedio una vez por semana o antes si las necesidades de la empresa lo requería, para favorecer a los plantadores y contratistas se tenían cláusulas en las que se establecía que si la compañía exportadora sin razones legales, no daba orden de cortar por lo menos una vez por semana, luego no podía rechazar el producto por madurez, sin embargo si el contratista no tenía el corte listo en el tiempo y hora indicado, se tomaría la producción de otro contratista para realizar esta labor, se estableció en los contratos para todo el Caribe que todos los días de la semana eran hábiles.

La Compañía podía pasar también los cables de teléfono y demás obras de infraestructura que requiriera por los predios de los contratistas sin pagar servidumbre $\mathrm{u}$ otro tipo de estipendio, el gobierno por su parte recibía un centavo oro americano por cada racimo de banano exportado.

Grafica 3. Precios cotizados por la United Fruit Co en New York por cargamentos de bananos producidos en Costa Rica en 1927

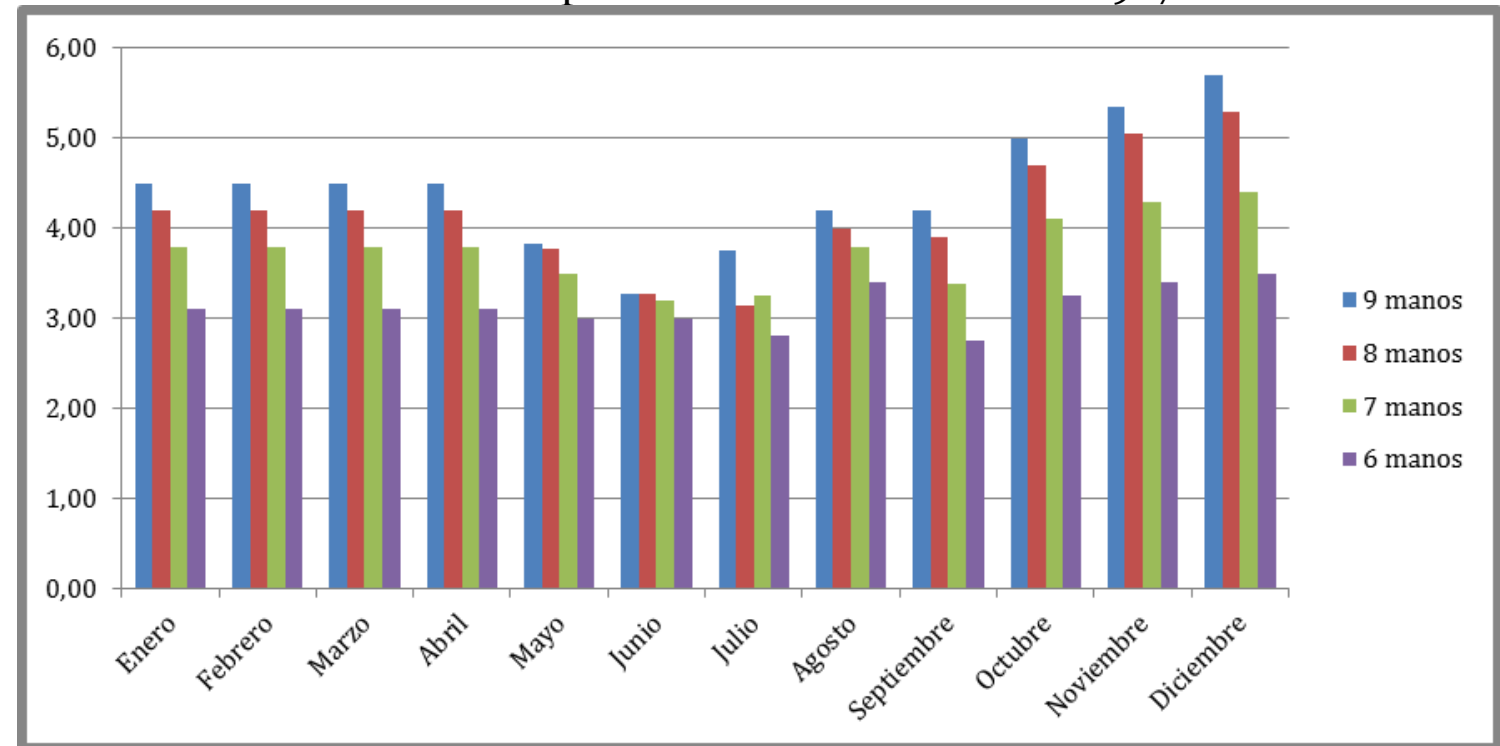

Fuente. Alfredo Saenz, The banana situation in the countries of de Caribbean Sea, pp.LV-LVI. Elaboración de la autora. 
El comportamiento de precios del año 27 difirió con relación a los años anteriores ya que en este año los racimos de 9 manos se pago en promedio a $\$ 4,5$, los de 8 manos a $\$ 4,0$, los de 7 por debajo de esta cifra y los de 6 por debajo de $\$ 3$ pesos oro americano, situación que indica la evidente caída de los precios con relación a los establecidos en los contratos.

Los contratos de arrendamiento de tierras, estaban controlados al igual que los de producción, por la Compañía, de tal manera que la tierra pertenecía a esta, la que había adquirido por compra directa a otras personas o simplemente y como sucedió en Colombia y en otros países, los gobiernos daba grandes extensiones de baldíos con el pretexto del desarrollo de la industria, situación que era contradictoria ya que en vez de entregar la propiedad de la tierra a los nacionales se le entregaba a la Compañía quien a su vez arrendaba la tierra a los nacionales productores bajo sus condiciones, una de ellas era que si el arrendatario dejaba de pagar por adelantado un mes, la Compañía podía inmediatamente tomar la posesión de las tierras incluyendo los cultivos y mejoras, en detrimento del arrendatario, el que debía pagar, aunque las condiciones climáticas o de producción le afectara. Además el contratista no podía subarrendar el terreno o sembrar otro tipo de cultivo.

Como ejemplo de ello tenemos que en Costa Rica Minor Keith, ${ }^{20}$ quien se hallaba vinculado con el ferrocarril, denunció hacía la década de 1890 por lo menos 170.00o hectáreas y la River Plate, $\mathbf{1 7 2 . 0 0 0}$ hectáreas, es decir entre ambos concentraban un total del $7 \%$ de la extensión actual de Costa Rica. ${ }^{21}$

En cuanto a la política de contratación de mano de obra ${ }^{22}$ se ajustó a los intereses de la Compañía y la política de pago por semanas o mes vencido, en Hon-

\footnotetext{
${ }^{20}$ Este empresario había iniciado el negocio del banano en varios países.

${ }^{21}$ Ronny Viales Hurtado, "La colonización agrícola de la Región Atlántica (Caribe) Costarricense entre 1870 y 1930 . El peso de la política agraria liberal y de las diversas formas de apropiación territorial", en Anuario de Estudios Centroamericanos vol.27, n. ${ }^{\circ}$, San José, Universidad de Costa Rica, 2001, pp.68-69. http://www.jstor.org/stable/25661350, Consultado: 15/o1/2013.

${ }^{22}$ Las luchas campesinas en América Latina, véase en Pablo González Casanova (coord.), Historia política de los campesinos latinoamericanos, vols.1 a 4, Madrid, Instituto de Investigaciones Sociales de la UNAM/Siglo Veintiuno Editores, 1998.
} 
duras por ejemplo se pagaba cada cuarenta y cinco días, expresados en los contratos que firmaban los trabajadores, en los cuales estos renunciaban al derecho que por cualquier ley se emitiera en lo sucesivo, a que se le pagara su salario o sueldo en otra fecha que no fuera la designada en este, cubría esta condición a los trabajadores directos, o por medio de contratistas. ${ }^{23}$

También fue punto común en las zonas bananeras, el hecho de que los trabajadores vivieran cerca de la compañía frutera y compraran en los comisariatos. Si el trabajador quería contar con dinero en efectivo debía perder no solamente el 50\% del valor de su ficha, sino que hasta podía perder el empleo. ${ }^{24}$ Estos puntos en comunes en cuanto a las políticas laborales, contratación y adquisición de tierras fueron establecidos por la United en todos los países en donde tenía presencia, por lo que sería interesante seguir con el análisis de la tendencia económica de dichas zonas bananeras. Esto sin contar que funcionaban como banco en las ciudades y zonas en donde se establecían.

Debido a los abusos y negligencia de los gobiernos locales se propuso la creación de la Liga Internacional de Bananeros, la que se encargaba de denunciar, no solamente la situación de los trabajadores, sino también de los agricultores, las desventajas para los países y las pocas utilidades que estos obtenían en este negocio, siendo la estrategia para establecerse en cada país la "amenaza permanente que se hacía de que si no se les daban los privilegios solicitados, se irían a otros países”, adicionalmente se denunció la adquisición de tierras limítrofes entre países, la compra de periódicos locales, así como la manipulación de diputados y senadores los que se convertían en sus representantes políticos y facilitadores de un marco jurídico favorable para sus intereses.

Esto condujo que hacía 1928 se formaran asociaciones de productores en Colombia, Costa Rica y Jamaica con el fin de competir con la United en la exportación del Banano, estos intentos se entendían como un esfuerzo adicional colaborativo entre los países productores para poner en practica la mencionada Liga, se

\footnotetext{
${ }^{23}$ Pablo González Casanova (coord.), Historia política de los campesinos latinoamericanos.

24 Vilma Laínez y Victor Meza, "El enclave bananero en la historia de Hoduras", en Anuario de Estudios Centroamericanos n. ${ }^{\circ}$, San José de Costa Rica, Universidad de Costa Rica. 1974, p. 218. http://www.jstor.org/stable/25661525. Consultado el 15/01/2013.
} 
aglutinaron alrededor de esta idea aquellas asociaciones costarricenses que en búsqueda de soluciones pacíficas escribieron una carta al presidente Hoover, en donde le ponían en conocimiento la miseria en la que estaban los países productores por los malos manejos de la United, enfatizando además, que en este proceso se perjudicaba a estos, pero también al pueblo norteamericano, ya que el banano que pagaban en 60 ctvs. lo vendían en sus mercados a tres o cuatro dólares. ${ }^{25}$

Los conflictos de tierras entre Guatemala y Honduras encontraron su origen en la apropiación de terrenos en los límites de estos países. Como se observa en el mapa 2, para el desarrollo de la producción en la zona bananera de Guatemala construyó 50 kilómetros de ferrocarril y dos puentes en la ribera derecha del Río Motagua el cual era soberanía de Honduras, de lo que podemos deducir que:

a) no respetaba las soberanías de los países,

b) solamente les interesaba ubicarse en los lugares en donde tenían mayor productividad indistintamente de las fronteras, se puede decir que no había un reconocimiento real de las fronteras entre países, estos se veían como un solo territorio,

c) y que en la medida en que se suscitaran conflictos seguían operando hasta tanto se solucionaran los mismo, sin embargo esta cumplía un doble papel, es decir, por un lado suscitaba el conflicto y por otro, su presencia misma no permitía que se le diera una solución adecuada, ya que cada país, ya sea por los intereses particulares o por la corrupción administrativa no estaban interesados en entrar en conflictos con la United.

${ }^{25}$ Alfredo Saenz, The banana situation in the countries of de Caribbean Sea, p.LXXXVII 


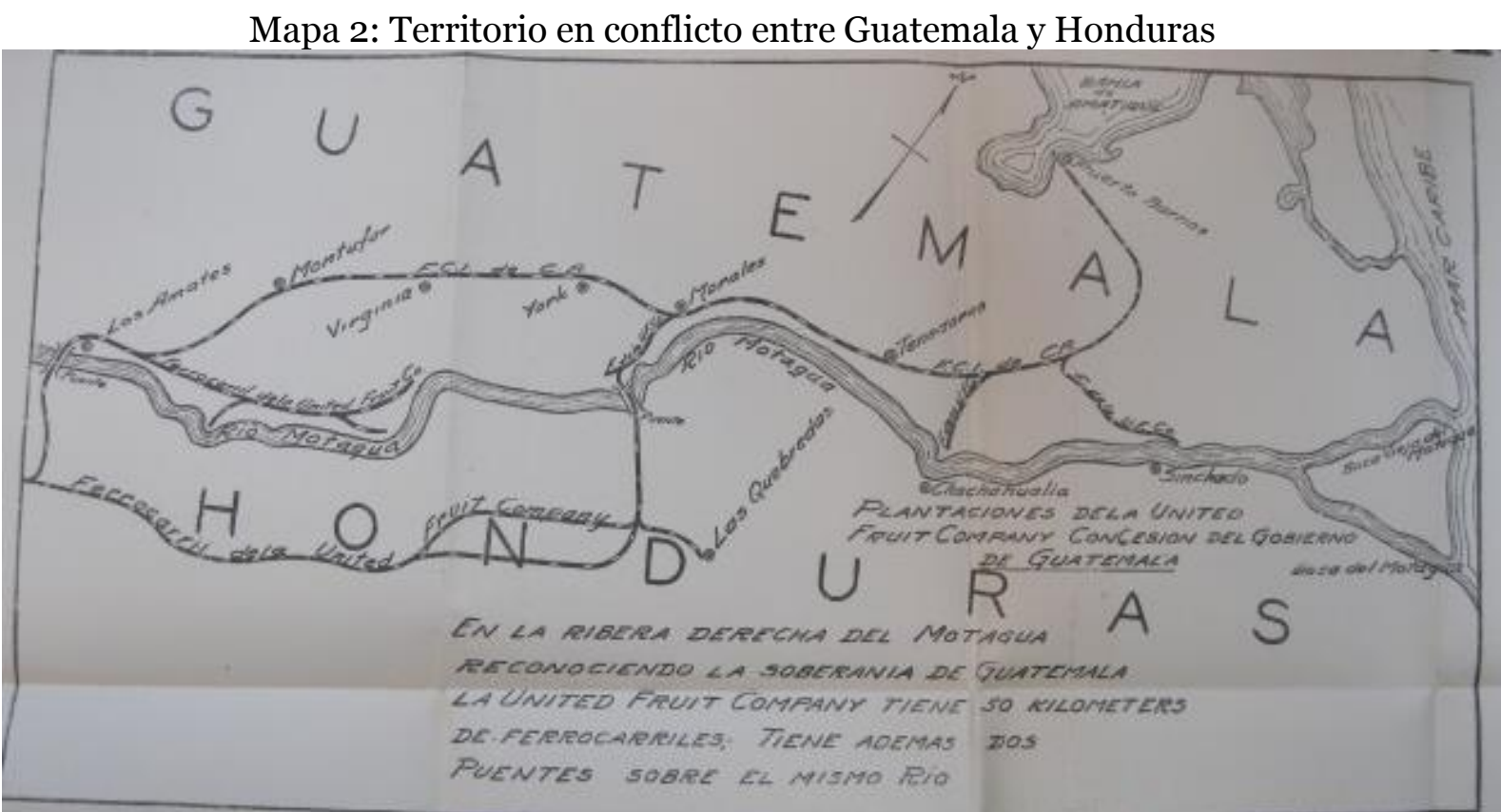

Fuente: Alfredo Saenz, The banana situation in the countries of de Caribbean Sea.

Con relación a Honduras en el mismo período estudiado, se inicio el comercio del banano a baja escala, sin embargo alcanzó a llegar a New York. La producción aún se hallaba en mano de pequeños productores hondureños, los que vendían a las compañías compradoras de banano, las cuales utilizaban miles de estrategias para bajar el precio en la compra y aumentar el precio de venta. Por su parte el gobierno de Honduras y ante el dinamismo del negocio, facilitó la concesión de terrenos para cultivos de banano y la celebración de contratos para la canalización de ríos, con el fin de que pudieran moverse mejor los barcos. ${ }^{26}$ La United Fruit Company trece años después de su fundación ya estaba instalada en Honduras, controlando el negocio por sí misma y a través de sus subsidiaras por concesiones hechas por el estado que la beneficiaba en muchos aspectos. En estas el trust quedaba libre del pago de derechos aduaneros y de toda clase de impuestos fiscales municipales, marítimos y terrestres, así como en maquinarias, rieles, carros, herramientas entre otros.

26 Vilma Laínez y Victor Meza, “El enclave bananero en la historia de Honduras”, pp.187-225. 
La concesión también les daba derecho a explotar los bosques nacionales, así como la cal, piedras que estuvieran en dichos terrenos ${ }^{27}$ de tal manera que en 1914 las tierras cultivadas por la United, de su propiedad, ascendía a quince mil acres, una década después ascendía a 87.800 acres, ${ }^{28}$ siendo el país más productor de banano en ese momento. ${ }^{29}$ La producción bananera fue tan importante en la economía del país que a comienzos del siglo xx, representaba el 53\% de todas las exportaciones. Si comparamos la producción bananera de Honduras con Colombia y Costa Rica, encontramos que entre los años de 1893 y 1900, se convirtió en el segundo productor, con una producción de racimos de 13.171.437.

Grafica 3: Comparativo entre Costa Rica, Honduras y Colombia en la producción de racimos de banano entre $1893-1900$

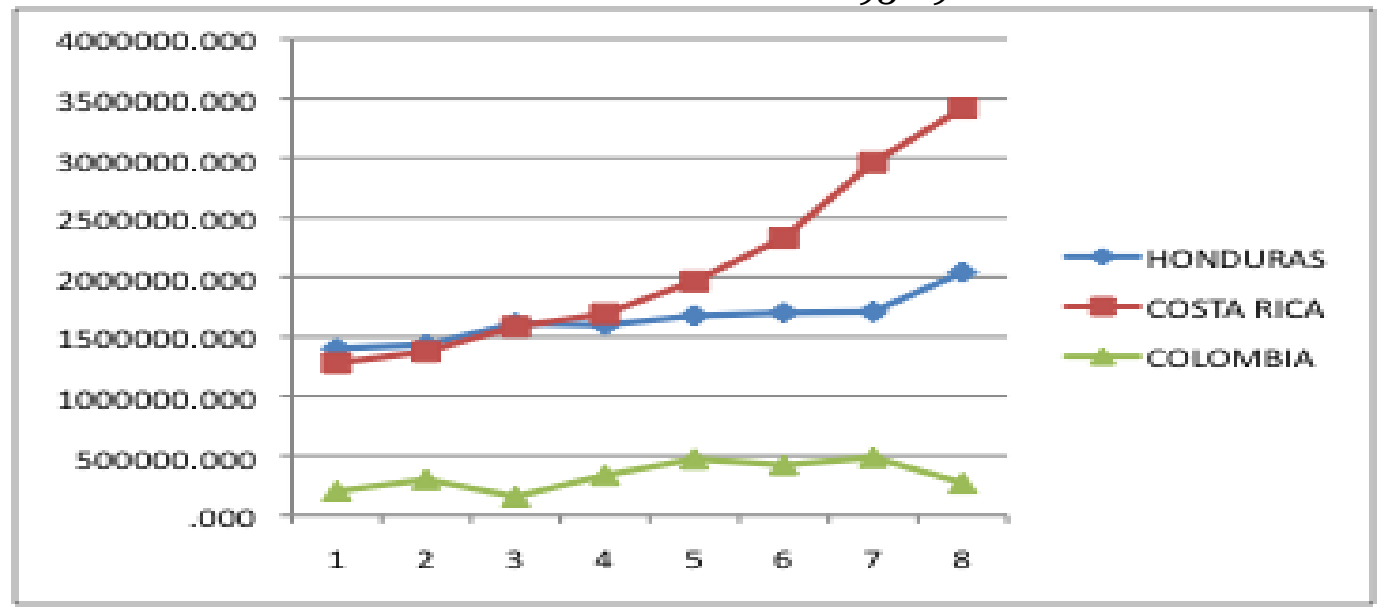

Fuente: Datos tomados de Vilma Laínez y Victor Meza, "El enclave bananero en la historia de Honduras", pp.187-225; Alfredo Saenz, The banana situation in the countries of de Caribbean Sea, pp.LIII-LIV; Adolfo Meisel, "Enfermedad Holandesa y exportaciones de banano en el caribe colombiano, 1910-1950”, p.18. Elaboración de la autora.

\footnotetext{
27 Vilma Laínez y Victor Meza, “El enclave bananero en la historia de Honduras”, pp.187-225.

28 Vilma Laínez y Victor Meza, “El enclave bananero en la historia de Honduras”, pp.187-225.

${ }^{29}$ Vilma Laínez y Victor Meza, “El enclave bananero en la historia de Honduras”, pp.187-225.
} 
Con relación a la deuda externa, no obstante el desarrollo bananero, hacía 1826 el $96 \%{ }^{30}$ de la deuda interna del gobierno era a favor de las compañías bananeras, prestamos causados para sostener y financiar las interminables revueltas armadas internas que aquejaban al país. ${ }^{31}$ De acuerdo con lo anterior, la estrategia tendía a establecer zonas bananeras en países simultáneos, lo que permitió que la United Fruit Company tuviera asegurada la producción. Si comparamos la producción de tres países de los más importantes en este negocio tenemos que la producción en Costa Rica entre el período de 1893 a 1900 superó, la producción de Honduras y Colombia, cuya producción en este intervalo fue bastante bajo con relación a los dos anteriores.

\section{Critica de la liga bananera y la posición de la United Fruit Company, frente a los conflictos e inconformidades suscitados en los países de América Latina por la producción bananera}

Frente a los problemas suscitados y a las voces de descontento de la mayoría de países de America Latina y el Caribe con relación a la forma como la United venía manejando el negocio del banano, en detrimento de la economía misma de estos países y sobre todo de los trabajadores $\mathrm{Mr}$ Cutter, ${ }^{32}$ se pronunció en el siguiente sentido:

\footnotetext{
${ }^{30}$ Según Laínez y Meza, la deuda en ese año ascendió a 7.763.549.64 pesos, de los que se adeudaban a las compañías bananeras en la siguiente relación:

Standard Fruit Company: 120.696,19

Cuyamel Fruit Company : 174.656,26

Tela y Truxillo RR. Co : $644.783,30$

N.Y. and Honduras Rosario Mining Company: 18.636,84

Zamuel Zemurray (Ferrocarril Nacional): 3.000.000,00

Santos Soto: 500.00,00

31 Vilma Laínez y Victor Meza, “El enclave bananero en la historia de Honduras”, pp.187-225.

32 Alfredo Saenz, The banana situation in the countries of de Caribbean Sea, pp.8-10.
} 
- Que el desarrollo comercial de América Latina a futuro asombraría al mundo.

- En la próxima década su progreso sería mayor que el alcanzado desde la conquista.

- La America Latina, era el mejor mercado natural de los estados Unidos.

- Critico lo que denominó la critica sin freno y propaganda de norteamericanos y europeos radicales, quienes se habían inventado el imperialismo norteamericano, por tanto la idea de las relaciones imperialistas entre Sur América y los Estados Unidos.

- Las ideas imperialistas no se tenían en cuenta cuando se establecían relaciones entre dos grandes zonas, una manufacturera y exportadora, y otra agrícola e importadora, que se necesitaban mutuamente.

- Además estos países necesitaban el capital de los inversionistas de los Estados Unidos.

- Y por último declaró, que estados Unidos debía hacer sus empréstitos a aquellos países en donde sus compañías industriales de fueran recibidas con Beneplácito.

Las anteriores observaciones giraron alrededor de las ideas económicas en las que primó la división de los países en productores de materias primas agrícolas, como era el caso de los de América Latina y el Caribe, los que a su vez por la falta de suficiente capital ${ }^{33}$ dependían de la inversión de capitales externos, tal como el caso del establecimiento de zonas bananeras, la primera de las cuales se desarrollo en Costa Rica, luego se estableció la Zona Bananera de Santa Marta, posteriormente Honduras y Guatemala, en todas estas Zonas Bananeras, el esquema de apropiación del ferrocarril, de tierras baldías y explotación de mano de obra fue el mismo, lo que indudablemente condujo a que los activos de la empresa pasaran de diez millones de dólares a 200,000 millones de dólares. ${ }^{34}$

\footnotetext{
$33 \mathrm{El}$ capital con que contaban los nacionales no era suficiente para establecer un ritmo de producción acorde con la dinámica del mercado internacional.

34 Vilma Laínez y Víctor Meza, “El enclave bananero en la historia de Honduras”, pp.187-225.
} 
Pero en esta confrontación no solamente se resaltaba la explotación hacía esta parte del continente, sino también la especulación del precio del banano a la población norteamericana, es decir la Liga, trascendió el discurso localista para analizar, la explotación de la United desde un punto de vista más global:

Esos doscientos millones de dólares de que tanto se ufana Mr. Cutter, amén de todos los centenares de millones de dólares que del haber latino y de los bolsillos del pueblo americano fueron a parar en gran parte a las insaciables arcas de los directores de aquella Compañía, han tenido `por origen, la extorsión del productor latino, la extorsión del erario nacional, puesto que prácticamente no paga ningún tributo, la extorsión del pueblo latino por medio del comisariato, el monopolio de los ferrocarriles construidos y pagados por estos países como en el caso de Casta Rica y Colombia, el monopolio del trasporte marítimo, la corrupción de nuestros hombres y; como un digno corolario, la explotación despiadada del pueblo noble americano [...]. ${ }^{35}$

Es decir, el problema no se veía como una política del Estado norteamericano, sino como la ambición desmedida de los inversionistas particulares, cuyo propósito giraba en torno a la optimización del capital invertido y a las ganancias que este reportara, esta actitud de la Liga, tal vez obedezca a una posición un poco consecuente con los Estados Unidos, pues es sabido que la inversión de capital, los empréstitos y la instalación de compañías productoras en America Latina se convirtió en la esperanza de progreso de dichos países, pues no se veía con malos ojos el capitalismo norteamericano, sino las prácticas de las United Fruit Company y sus múltiples filiales.

Sin embargo, hay que tener en cuenta que la United Fruit Company también creo problemas en el comercio del banano en Estados Unidos, ya que para controlar su comercio y establecerse como monopolio, compró negocios y propiedades de sus competidores con el fin de controlar y regular la venta en ese país. Sus acciones por el control total de la fruta fueron más allá, es decir sabotear a los competidores como se puede leer en la demanda que hizo en 1903 la American Banana Company

${ }^{35}$ Alfredo Saenz, The banana situation in the countries of de Caribbean Sea, pp.15-16. 
contra la United Fruit Compani, ${ }^{36}$ en donde el demandante pone de manifiesto los métodos que el acusado había llevado a cabo para arruinarles su negocio, en los países productores de banano y en el control del precio en el mercado.

\section{Ideas concluyentes}

Para finalizar tenemos que la política de la United Fruit Company, trust norteamericano pregonaba que su instauración en los países de America Latina y el Caribe se debía básicamente al derecho que estos tenían de desarrollarse y que ofrecía las estrategias pertinentes para que esto sucediera, sobre todo en el desarrollo de obras de infraestructura de vías férreas y caminos, sin embargo la practica estaba muy lejos del discurso, pues la forma como desarrollaron el negocio solamente dejó en las zonas de producción conflictos sociales, atraso económico y devastación del terreno, además de una mayor corrupción política y administrativa al nivel local.

De otra parte, los agricultores nacionales quedaron en calidad generalmente de arrendatarios y amarrados a contratos tendientes a su ruina, mientras los trabajadores se veían más como máquinas de producción, por lo que sus condiciones de contratación y salubridad ni siquiera se acercaban a las primeras normas regulatorias del trabajo, como fue el caso de Colombia.

El sistema de vales y la obligación de los trabajadores de consumir y comprar lo que las empresas filiales producían, condujo a los conflictos ampliamente conocidos en todo lo que fue las zonas bananeras. La intromisión en los asuntos internos de los países, sobre todo en los que tenían que ver con las fronteras y el fortalecimiento del capital de la United son muestras evidentes de la explotación desmedida de materias primas con el beneplácito de los gobiernos locales y del gobierno de los Estados Unidos. No obstante lo anterior, en la crisis del 29 que des-

\footnotetext{
${ }^{36}$ Esta demanda con las sentencias correspondientes puede leerse en American Society of Internacional Law, American Banana Company v. United Fruit Company vol.3, n. ${ }^{\circ}$ 4, 1909, pp.1006-1011. http://jstor.org/stable/2186436. Consultado 15/01/2013
} 
encadenó un nuevo rumbo en las políticas económicas, los países de la región fueron llamados a ocupar otro papel en la economía mundial, el cual todavía en la actualidad no es lo suficientemente claro.

\section{Bibliografía:}

American Society of Internacional Law, American Banana Company v. United Fruit Company vol.3, n. ${ }^{\circ}$, 1909, pp.1006-1011. http://jstor.org/stable/2186436. Consultado 15/01/2013

Arango Zuluaga, Carlos, Sobrevivientes de las bananeras, Bogotá, Ecoe, 1985.

Archila Neira, Mauricio, "Masacre de las bananeras: diciembre 6 de 1928”, en $R e$ vista Credencial Historia n. ${ }^{\circ} 117$, Bogotá, 1999.

Bauer, Arnold, "La Hispanoamérica rural, 1870-1930", en Historia económica de América Latina, Madrid, Crítica, 2002.

Bejarano Ávila, Jesús Antonio, "El despegue cafetero (1900-1928)", en Historia económica de Colombia, Bogotá, Editorial Planeta, 2007, pp.195-232.

Botero, Fernando, "El enclave agrícola en la zona bananera de Santa Marta", en Cuadernos colombianos vol.3, n. ${ }^{\circ} 11$, Medellín, 1977, pp.312-389.

Bucheli, Marcelo "Enforcing Business Contracts in South America: The United Fruit Company and Colombian Banana Planters in the Twentieth Century. The President and Fellows of Harvard College", in The Business History Review vol.78, n. ${ }^{\circ}$ 2, 2004, pp.181-212. www.jstor.org/stable/25661863. Consultado 15/01/2013.

Brungardt, Philip Maurice, "La United Fruit Company en Colombia”, en Innovar n. ${ }^{\circ}$, Bogotá, Universidad Nacional, 1995, pp.107-118.

González Casanova Pablo (coord.), Historia política de los campesinos latinoamericanos, vols.1 a 4, Madrid, Instituto de Investigaciones Sociales de la UNAM/Siglo Veintiuno Editores, 1998. 
Elías Caro Jorge Enrique, "El puerto de Santa Marta: determinantes de crecimiento y desarrollo de una ciudad caribeña (1810-1860)", en Ciudades portuarios en la Gran Cuenca del Caribe, Visión histórica, Barranquilla, Universidad del Norte/Universidad del Magdalena, 2010, pp.328-354.

Elías Caro Jorge, "La masacre obrera de 1928 en la zona bananera del Magdalena - Colombia”, en Andes vol.22, n. ${ }^{\circ}$, Salta, Universidad Nacional de Salta, 2011.

Glade William, “América Latina y la economía internacional, 1870-1914”, en Historia económica de América latina, Madrid, Crítica, 2002.

Kalmanovitz, Salomón y López Enciso, Enrique, La agricultura Colombiana en el siglo XIX, Bogotá, Fondo de Cultura Económica, 2006.

Tovar Zambrano, Bernardo, La intervención económica del Estado en Colombia, 1914-1936, Bogotá Banco Popular, 1984.

Laínez, Vilma y Meza, Víctor, "El enclave bananero en la historia de Hoduras", en Anuario de Estudios Centroamericanos n. ${ }^{\circ}$, San José de Costa Rica, Universidad de Costa Rica. 1974, p.218. http://www.jstor.org/stable/25661525. Consultado el 15/01/2013.

Legrand Catherine, "Living in Macondo. Economy and culture in a United Fruit Company, banana enclave in Colombia", in Close encounters of Empire: writing the cultural history of U.s. Latin American relations, Durham, Duke University Press, 1998.

López Garavito, Luis Fernando, Historia de la Hacienda y el tesoro en Colombia, 1821-1900, Bogotá, Banco de la República, 1992.

Meisel Roca, Adolfo, "Enfermedad Holandesa y exportaciones de banano en el caribe colombiano, 1910-1950", en Cuaderno de Historia económica y Empresarial, Cartagena, Banco de la República, 2010, pp.1-56.

Murillo Jiménez, Hugo et al, "Las relaciones comerciales entre Costa Rica y los Estados Unidos a través de los informes consulares, 1880-1901”, en Anuario 
de Estudios Centroamericanos vol.13, n. ${ }^{\circ}$, San José de Costa Rica, Universidad de Costa Rica, 1978, pp.135-136.

Quesada Monge, Rodrigo, "Ferrocarriles y crecimiento económico: el caso de la Costa Rica Railway Company, 1871-1905”, en Anuario de Estudios Centroamericanos vol.9, San José de Costa Rica, Universidad de Costa Rica, pp.87-119. www.jstor.org/stable/25661863. Consultado 15/01/2013.

Reyes, Rafael, Conferencia leída el 19 de abril de 1908 en el Salón de Grados, Colombia, Imprenta Nacional, Bogotá, 1908.

Saenz, Alfredo, The banana situatiotion in the countries of de Caribbean Sea, San José de Costa Rica, Imprenta Borrase Hermanos1928.

Viales Hurtado, Ronny, "La colonización agrícola de la Región Atlántica (Caribe) Costarricense entre 1870 y 1930. El peso de la política agraria liberal y de las diversas formas de apropiación territorial”, en Anuario de Estudios Centroamericanos vol.27, n. ${ }^{\circ}$, San José, Universidad de Costa Rica, 2001, pp.57-100. http://www.jstor.org/stable/25661350, Consultado: 15/01/2013. 